\title{
Labyrinthe
}

$30 \mid 2008$ (2)

Ecologie $=X$

\section{La recherche écologique à l'épreuve de la philosophie de l'environnement}

\section{Julien Delord}

\section{Q OpenEdition}

\section{Journals}

Édition électronique

URL : https://journals.openedition.org/labyrinthe/3773

DOI : $10.4000 /$ labyrinthe.3773

ISSN : 1950-6031

Éditeur

Hermann

Édition imprimée

Date de publication : 10 juin 2008

Pagination : 74-88

ISBN : 978-2-9526131-7-0

\section{Référence électronique}

Julien Delord, « La recherche écologique à l'épreuve de la philosophie de l'environnement », Labyrinthe [En ligne], 30 | 2008 (2), mis en ligne le 27 mai 2008, consulté le 12 janvier 2022. URL : http:// journals.openedition.org/labyrinthe/3773; DOI : https://doi.org/10.4000/labyrinthe.3773 


\title{
LA RECHERCHE ÉCOLOGIQUE À L'ÉPREUVE DE LA PHILOSOPHIE DE L'ENVIRONNEMENT
}

\author{
Julien DELORD \\ julio19fr@yahoo.fr
}

L'éthique de l'environnement n'échappe pas à une critique fondamentale qui s'adresse à l'ensemble des éthiques dites «appliquées » qui ont fleuri ces dernières décennies, qu'il s'agisse de la bioéthique ou de l'éthique économique: elles témoignent toutes, à divers degrés, d'une insigne faiblesse normative. Par leur nature circonstanciée, ouverte, exploratoire, voire subjective, par leur volonté assumée de dépasser les cadres contraignants mais puissants de l'ancienne morale judéo-chrétienne sécularisée, elles gagnent en adéquation descriptive et en originalité conceptuelle ce qu'elles perdent en force normative. L'impuissance (apparente) de l'éthique environnementale à résoudre les problèmes environnementaux et à contrer les instincts destructeurs de l'humanité sur les espèces, les écosystèmes et par extension sur la totalité des conditions de survie de l'espèce humaine, devrait constituer une priorité de réflexion pour tout philosophe de l'environnement engagél. Comment dès lors penser, afin de le promouvoir, le passage des valeurs définies par l'éthique environnementale aux attitudes et aux actions concrètes envers l'environnement? Si la réponse à cette question intéresse le «citoyen" soucieux d'agir selon des normes qui garantissent la protection de son environnement $\rightarrow$ "Quelle éthique pour l'écologie ? » $\leftarrow$, elle n'intéresse pas moins le «scientifique», l'écologue, dont la praxis est en soi dirigée vers un milieu dont il étudie les interactions avec les êtres vivants - éventuellement y compris lui-même - et qui fonde en raison son activité de chercheur. L'écologie scientifique apparaît donc comme un terrain d'étude privilégié où agissent quotidiennement des acteurs

\footnotetext{
1. Voir Barnabas Dickson, «The Ethicist Conception of Environmental Problems», Environmental Values, vol. 9, $\mathrm{n}^{\circ}$ 2, 2000, p. 127-152; Kassiola, « Can environmental ethics "solve" environmental problems and save the world? Yes, but first we must recognise the essential normative nature of environmental problems n, Environmental Values, vol. 12, n 4, 2003, p. 489-514.
} 
très bien informés des problèmes environnementaux et supposés réactifs face aux nouveaux enjeux écologiques, scientifiques et sociaux ${ }^{2}$. Les transformations récentes concernant la nature des objets, des méthodes et des structures d'organisation de la recherche en écologie scientifique témoignent-elles alors de la prégnance des valeurs environnementales? Et si oui, sous quelles formes?

\section{Une éthique de l'expérimentation écologique}

Dans la mesure où les recherches écologiques n'ont pas seulement pour objet de nous donner à comprendre l'environnement, mais que beaucoup d'entre elles, et en particulier celles qui relèvent de la biologie de la conservation, interagissent avec différents éléments des écosystèmes sauvages, parfois très fragiles, il est important de rendre compte de l'intégration par les écologues eux-mêmes des enjeux de l'éthique environnementale dans leurs activités professionnelles.

Car il existe un conflit manifeste entre certaines normes de l'éthique de la connaissance et l'éthique de l'environnement. L'écologie, comme l'a remarqué Bruno Latour, «prétend protéger la nature et la mettre à l'abri de l'homme, mais, dans tous les cas, cela revient à inclure davantage les humains qui interviennent encore plus souvent, de façon encore plus fine, encore plus intime et avec un appareillage scientifique encore plus envahissant ${ }^{3} »$. L'activité scientifique requiert en effet d'interagir, et parfois de façon brutale, avec le système étudié. En retour, l'objet d'étude rétroagit aux perturbations qu'il a subies et modifie son fonctionnement normal. Des interactions mal maîtrisées entre le scientifique et son objet d'étude conduisent en fin de compte à une situation "perdant-perdant»: le scientifique perd sur le plan des valeurs épistémiques (précision, vérité, etc.), et les entités environnementales sur le plan de leurs «intérêts », voire de leurs droits à ne pas être détruites ou altérées sans raison.

2. Je n'ai pas pour objectif d'ouvrir un nouveau débat sur les limites entre faits et valeurs, sur la nature des vérités scientifiques, sur l'ambiguîté de la fonction sociale du chercheur (scientifique pur, expert, conseiller du prince, citoyen éclairé, etc.), même s'il est peu contestable qu'il s'agisse là d'ambigunttés qui rendent possible la dynamique «éthique » de la science.

3. Politiques de la nature, Paris, La Découverte, 1999, p. 35. 
Ne mettons-nous pas, dans certains cas, les espèces menacées plus en danger en essayant de les connaître? N'attribuons-nous pas un poids excessif au principe que la connaissance constitue nécessairement un bien, ou plutôt qu'un progrès des connaissances est souhaitable en toute situation? Ces dernières années, de nombreux écologistes, parmi lesquels sir Robert May, se sont inquiétés de l'impact négatif que pouvait avoir la recherche écologique sur des populations fragiles ${ }^{4}$. De son côté, Michel Gauthier-Clerc a montré que le marquage des manchots dans le cadre du suivi de leurs déplacements avait un effet direct sur leurs chances de survie et de reproduction, sans parler du biais que cet effet entraînait sur les résultats des enquêtes scientifiques ${ }^{5}$.

Ce problème éthique n'est pas tant inquiétant par l'ampleur (somme toute limitée) des conséquences écologiques des activités de recherche en question, que par la révélation de l'existence symptomatique d'un décalage préoccupant entre l'activité de recherche et son objet qui en constitue ici le contexte. Le précédent bioéthique nous avait révélé les interrogations, voire les dérives d'une recherche biomédicale qui, à force de réduire le corps humain à une somme d'organes, de tissus et de molécules, avait fini par occulter l'homme dans sa vérité d'être vivant, sensible, social et pensant. Certains écologistes ont-ils peut-être oublié, à force d'étudier l'environnement seulement en terme de systèmes écologiques et de dynamiques de populations, que ces systèmes fort complexes étaient avant tout constitués d'organismes vivants, parfois fragiles, en interaction?

La nécessaire prise en compte d'une éthique de l'environnement, même a minima, dans la définition des méthodes de recherche impose aux scientifiques un retour réflexif à bien des égards salutaire; il y a sans aucun doute dans le respect de l'environnement une dimension déontologique, au sens de régulation morale de la profession de chercheur, qui ne doit pas voiler la dimension pragmatique (au sens philosophique) de ces valeurs environnementales contextuelles. Le pragmatisme, au sens des théories épistémologiques, s'entend comme la poursuite, par alternance d'expériences et de théorisations dans un environnement déterminé, d'un idéal de vérité conçu comme horizon asymp-

\footnotetext{
4. Voir Robert M. May, «Ethics and Amphibians n, Nature, vol. 431, $n^{\circ}$ 7007, 2004, p.403; John R. Spear, « Minimum Impact Research », Conservation Biology, vol. 18, 2004, p. 86.

5. Michel Gauthier-Clerc et alii., « Long-term Effects of Flipper Bands on Penguins n, Proceedings of the Royal Society of London B, vol. 10, 2004, p. 1098.
} 
totique. La poursuite de la vérité est ainsi comparable à un processus évolutif où l'objet, la méthode et l'environnement de recherche se modifient et doivent s'adapter mutuellement. Toute recherche portant sur des espèces affectées en retour par cette même recherche nécessite l'adoption de méthodes nouvelles conduisant à des résultats inédits, plus adéquats donc plus vrais, au fur et à mesure d'un processus adaptatif sans fin.

On constate ainsi qu'un ensemble de principes éthiques externes, ou plutôt contextuels par rapport à l'activité de recherche ("Aucun être vivant ne doit pas souffrir inutilement», «Il ne faut pas perturber le déroulement d'un processus biologique unique: la marche des manchots empereurs", etc.), pénètrent au cœur même de son fonctionnement afin de la modifier, et cela à partir de considérations pragmatiques qui impliquent une circulation permanente des savoirs et des représentations entre l' "environnement-contexte" et l' « environnement-objet». Cette perspective pose évidemment la question de la capacité de l'homme à comprendre la nature sans la détruire en partie, destruction qui est la rançon du canon analytique et réductionniste triomphant. Peut-être faut-il méditer cet aphorisme nihiliste de Nietzsche («Nous préférons tous la destruction de l'humanité à la régression de la connaissance ${ }^{6}$ !») qui s'interrogeait sur notre capacité, nous Occidentaux, à vivre dans un monde qu'on ne peut connaître parfaitement.

\section{La biologie de la conservation et les nouveaux objets de l'écologie}

La biologie (ou écologie) de la conservation (BC) fournit un exemple, plus visible encore, de l'impact de l'éthique sur les sciences écologiques. Cette science pluridisciplinaire, à la croisée des sciences humaines et de la biologie, est née en Europe et aux États-Unis dès la fin des années 1960, à une époque relativement contemporaine de l'émergence des mouvements de contestation dans lesquels s'incrit la naissance de l'environmental ethics $\rightarrow$ «De Frederick J. Turner à Donald Worster " $\leftarrow$, du souci de certains écologistes d'appuyer scientifiquement les efforts en vue de la conservation des espèces et des écosystèmes

6. Friedrich Nietzsche, Aurore, Paris, Gallimard, 1989 [1881]. 
menacés par la crise environnementale. La BC constituerait ainsi une sorte de clinique et de thérapeutique au service de la santé d'un environnement malade de l'homme. Or, on pourrait lui rapporter cette analyse de Canguilhem à propos du champ médical :

La clinique n'est pas une science et ne sera jamais une science, alors même qu'elle usera de moyens à efficacité toujours plus scientifiquement garantie. La clinique ne se sépare pas de la thérapeutique et la thérapeutique est une technique d'instauration ou de restauration du normal, dont la fin $[\ldots]$ échappe à la juridiction du savoir objectif?

La BC s'articule elle aussi autour de fins qui enjoignent de préserver la biodiversité et de rétablir les normes de fonctionnement des écosystèmes, ce que l'on nomme aujourd'hui les «services écosystémiques ». Les valeurs ainsi attribuées à la biodiversité peuvent être toutes perçues comme étant constitutives de la $\mathrm{BC}$, car elles représentent la raison d'être de son existence. Les biologistes de la conservation sont dès lors incités à se faire les avocats des valeurs propres à leur objet d'étude 8 . Pour Michael Soulé, l'éthique est au cœur même de la conservation; loin d'une neutralité axiologique abstraite qui constituerait l'idéal des sciences fondamentales, la $\mathrm{BC}$ doit constamment réaffirmer sa nature évaluative. Elle n'est pas simplement descriptive, elle est normative:

La diversité des organismes est bonne. [...] La complexité écologique est bonne. [...] L'évolution est bonne. [...] La diversité biotique a une valeur intrinsèque ${ }^{9}$.

Mieux encore, la BC est une science de crise qui, selon Michael Soulé, est un peu à l'écologie ce que la chirurgie est à la physiologie, ou ce que la biologie du cancer est à la biologie générale. Comme dans toute discipline de crise, le temps est un facteur limitant crucial, la problématisation souvent incomplète, l'analyse de la situation superficielle et les conclusions rapides - ce qui implique de tolérer un degré d'incertitude parfois élevé. Il s'agit à bien des égards d'une science en par-

7. Georges Canguilhem, Le Normal et le Pathologique, Paris, PUF, 1966, p. 153.

8. Jay Odenbaugh, « Values, Advocacy and Conservation Biology », Environmental Values, vol. 12. $n^{\circ} 1,2003$, p. 55-69.

9. Michael Soule, « What Is Conservation Biology? n, BioScience, vol. 35, n 11, 1985, p. 727-34. 
tie «post-normale ${ }^{10}$ », qui délaisse le mythe d'une science transcendante oublieuse des dispositifs techniques qui la rendent possible.

Sous l'influence de ces enjeux éthiques, la BC s'est donné un ensemble d'objets d'étude qui se démarquent en partie des entités écologiques servant de «modèle» aux expérimentateurs et aux théoriciens de l'écologie générale. Graham Caughley a ainsi distingué deux approches "paradigmatiques» au sein de la BC pour ce qui est de l'étude de la biodiversité en danger: le paradigme des populations déclinantes et le paradigme des petites populations ${ }^{11}$. Ces deux modèles témoignent de l'originalité d'une discipline conduite à explorer des champs délaissés initialement par l'écologie théorique: un exemple éclairant de ce décalage épistémique nous est fourni par les biologistes des populations qui ont toujours privilégié les phases de croissance des populations dans l'étude de leurs dynamiques démographiques. Ainsi, un manuel célèbre d'écologie ${ }^{12}$ publié en 1949 consacrait-il trois fois plus de pages à l'étude des phases de croissance et d'équilibre des populations qu'à celle des phases de décroissance. Par ailleurs, pour diverses raisons, liées en particulier à une emphase importante sur la notion de généralité dans la détermination des lois et des règles écologiques, les écologistes et les biologistes de l'évolution ont longtemps privilégié l'étude des grandes populations où n'interviennent pas (ou de manière négligeable) les interférences limites provoquées par une démographie réduite.

De manière fondamentale, c'est la notion même de «biodiversité», forgée par Peter Rosen et Edward O. Wilson en 1985, qui est devenue un enjeu épistémologique âprement débattu et revendiqué. Il n'est sans doute pas exagéré d'affirmer qu'il s'agit d'un exemple typique de concept «construit socialement» dans la mesure où il est sans référent réel univoque: parle-t-on d'entités, de structures ou de processus divers? De diversités génétiques, phylogénétiques, morphologiques, écologiques, etc.? Peut-on agréger les diversités propres à des niveaux hiérarchiques distincts? Il est indéniable toutefois que l'idée de biodiversité met en lumière, dans sa référence à la pluralité et à l'altérité de la nature, toute la valeur d'un objet kaléidoscopique sensible aux destructions humaines.

10. Silvio Funtowicz et Jerry Ravetz, «Science for the Post-Normal Age », Futures, vol. 25, ${ }^{\circ} 7$, 1993, p. 735-755.

11. Graeme Caughley, « Directions in Conservation Biology », Journal of Animal Ecology, vol. 63, 1994, p. 215-244.

12. Warder C. Allee et alii, Principles of Animal Ecology, Philadelphie, W.B. Saunders, 1949. 
Le choix des objets d'étude, clairement influencé par la dimension engagée de la $\mathrm{BC}$, est (en partie au moins) la résultante d'une décision éthique et pas seulement d'un intérêt épistémique. La dimension pragmatique - dans un sens non technique du terme - de ce choix doit nous conduire à reconsidérer les critères de sélection des objets «bon à penser» en science, qui tiennent manifestement à de nombreuses fécondations intellectuelles croisées entre intérêts, contraintes, et imagination normative.

\section{Une science plus «juste»: l'écologie participative}

L'influence de l'éthique sur la science ne se limite pas à la dynamique interne de celle-ci, mais concerne aussi son organisation et sa relation avec l'ensemble de la société. L'éthique environnementale proposerait ainsi un troisième type de valeurs que je qualifierais d' «interactionnelles " au sens où elles naîtraient de l'interaction des humains au sein et en vue d'un environnement déterminé; mais encore faut-il que l'interaction soit constructive, et pour cela «juste» ou plutôt «équitable» dans le sens rawlsien envisagé ci-dessous. Car n'oublions pas que la crise environnementale se double d'une crise des rapports «science-société».

À la suite des scandales sanitaires et environnementaux à répétition de la décennie, les institutions de recherche ont été confrontées à une contestation croissante des prérogatives des experts et des chercheurs détenteurs de savoirs «officiels». Comment recréer de nouveaux liens entre un savoir en train de se faire (la recherche) et une société inquiète d'être soumise à des techniques néfastes et incontrôlables? Ces expériences de (re)prise de contact et d'échanges constructifs de points de vue entre scientifiques et citoyens ont pris diverses formes (conférences citoyens, création de "science shops", sciences participatives ou sciences citoyennes) qui impliquent toutes à des degrés divers la coconstruction des problématiques et le partage des résultats scientifiques $^{13}$.

13. Voir Michel Callon et alii, Agir dans un monde incertain, Paris, Seuil, 2001. Le seul véritable exemple de conférence citoyenne en France date de 1998 avec la conférence « OGM ». Les autres dispositifs se résument le plus souvent à une simple « consultation » des citoyens. 
On peut parier que l'implication de plus en plus assumée des écologues professionnels dans les questions de protection et de gestion de l'environnement les a conduits à modifier leur position de savants prétendument «neutres» revendiquée jusque-là, pour devenir des experts. Sans pour autant se transformer tous en «lanceurs d'alertes", les écologistes professionnels sont devenus plus réactifs face aux problèmes environnementaux. Dans le contexte français, cela doit également être remis en perspective: il existe en effet historiquement une distance soupçonneuse entre écologistes et naturalistes, incarnant parfois jusqu'à la caricature les oppositions «professionnels» et «amateurs», «théorie» et "pratique», «élite» et "peuple». En se rapprochant des positions des naturalistes amateurs, les scientifiques professionnels leur reconnaissent désormais le rôle d'interlocuteurs privilégiés dans la mise en place de projets scientifiques à grande échelle, comme la collecte de données ou la surveillance de milieux particuliers.

Pour illustrer ce fait nouveau, je prendrai comme exemple le programme «Suivi temporaire des oiseaux communs» (STOC) de collecte de données relatives aux populations d'oiseaux sur le territoire français, mis en place par le Centre de recherche sur la biologie des populations d'oiseaux (CRBPO) du Muséum national d'histoire naturelle. Ce projet fait partie d'un programme plus large, depuis la fin des années 1990, de surveillance et de suivi de la biodiversité en France (Vigie-Nature) qui concerne les papillons de jardin, les chauves-souris, et de nombreuses plantes.

L'objectif de l'analyse qui suit n'est pas de mettre au jour les mécanismes sociaux, institutionnels et épistémiques de mise en place et de fonctionnement de cet exemple réussi de science participative ${ }^{14}$, mais d'analyser sous l'angle éthique ses présupposés en termes de «justice environnementale». Cette forme de justice traite traditionnellement de la distribution égale ou équitable, entre humains, des biens, des services, des ressources ainsi que des risques et des menaces issues de l'environnement ${ }^{15}$. On peut aussi inclure les questions du partage équitable des investissements et des bénéfices environnementaux tout comme l'accès démocratique à l'information et à la participation dans

14. Voir l'analyse très pertinente de Harold Levrel, « Construire des indicateurs durables à partir d'un savoir issu de multiples pratiques: le cas de la biodiversité „, Gérer et Comprendre, $\mathrm{n}^{\circ} 85$, septembre 2006, p. 51-62.

15. Voir Peter Wenz, Environmental Justice, Albany, State University of New York Press, 1988. 
les processus de décision concernant l'environnement. Elle ne doit pas être confondue avec l'idée de "justice écologique ${ }^{16}$ » qui soutient que les entités écologiques en tant que telles (animaux, plantes, espèces, etc.) possèdent certains types de droits obligeant les humains.

La France s'est engagée, dans le cadre de programmes européens qui prolongent la Convention sur la diversité biologique signée à Rio en 1992, à stopper sa perte de biodiversité d'ici à 2010. Il lui faut pour cela disposer d'indicateurs fiables qui reflètent le plus finement possible l'évolution de cette biodiversité. Le propre d'un indicateur par rapport à une mesure est de fournir une information de qualité (scientifiquement validée) qui réponde à des besoins pratiques (politiquement utile, gérable, compréhensible, simple). À cette aune, l'indicateur basé sur le simple nombre d'espèces qui s'éteignent ou en danger d'extinction est certes instructif et facile à comprendre, mais il est, d'une part, assez grossier et, d'autre part, ne permet guère d'anticiper ou d'agir préventivement sur les causes de perte de la biodiversité. L'indicateur STOC, au lieu de rendre compte de la richesse aviaire en espèces d'un habitat donné (ce vers quoi tendait traditionnellement l'activité des ornithologues), donne une estimation de l'abondance des différentes espèces suivies au cours du temps. Autrement dit, ce sont la taille et le nombre des populations de chaque espèce qui vont être estimés et non plus une information binaire de type "présence/absence».

En conséquence, le protocole de relevé des données est à la fois plus complexe et plus lourd à organiser pour le CRBPO. Sans la participation de centaines d'ornithologues amateurs et bénévoles, ce travail aurait été financièrement impossible à réaliser. Pourtant, ce même protocole, qui implique un tirage aléatoire des sites d'écoute et une présence de seulement cinq minutes sur le site à plusieurs semaines d'intervalle, constitue une pratique inédite, voire même incongrue, pour un ornithologue amateur. Et ce ne sont certainement pas les maigres avantages matériels (dons de filets pour les associations d'ornithologues bagueurs par exemple) qui expliquent la collaboration active des naturalistes amateurs.

Il y a clairement une dynamique sociologique dont il faudra analyser les ressorts normatifs sous-jacents. Derrière la (re)connaissance des compétences et du rôle des naturalistes amateurs par les écologistes et

16. Voir Brian Baxter, A Theory of Ecological Justice, New York, Routledge, 2004. 
biologistes de la conservation professionnels, du changement de perception des anciens «intégristes de la conservation, sans culture scientifique, des premiers temps ${ }^{17}$ », ou encore des «doux rêveurs chevelus des débuts ${ }^{18}$ ", se cache sans aucun doute le fait que nombre de jeunes naturalistes impliqués ou travaillant dans les associations possèdent désormais une formation approfondie en sciences de l'environnement ${ }^{19}:$ « Les grandes associations comme le WWF ou l'UICN, affirme Robert Barbault, ont lu la littérature (scientifique). En plus, elles disposent d'un recul, d'une stratégie que les scientifiques, souvent très individualistes, n'ont $\operatorname{pas}^{20}$ ». De l'autre côté, on a observé un regain d'intérêt des associations naturalistes envers les scientifiques. Comme l'avait montré Denis Buhot au début des années 1980 à propos de l'«Association pour la protection des rapaces", ses membres faisaient très majoritairement partie d'une classe moyenne intellectuelle provinciale, qu'il décrivait lui-même comme frustrée économiquement et socialement, et qui se réfugiait derrière une éthique intégriste de la conservation, revendiquant la pureté de l'amateurisme, des pratiques de terrain et du régionalisme contre toutes les «compromissions" gestionnaires, médiatiques et politiques des scientifiques "parisiens ${ }^{21}$. De nombreux facteurs, dont la mise en réseau des connaissances et des expériences locales grâce à Internet, le besoin de reconnaissance de ces associations au niveau local et la volonté de collaborer avec des gestionnaires de la nature de plus en plus nombreux, ont pu servir de motivation pour établir des liens plus étroits avec des scientifiques (liens qui ont toujours existé avec le Muséum, mais sur un mode plus fortement hiérarchisé et restreint aux «initiés»).

Cette forme de mobilisation nouvelle, réticulée, pluridisciplinaire, entre scientifiques et naturalistes, parie sur l'intelligence distribuée des naturalistes amateurs de terrain; elle signe aussi l'émergence d'une science plus modeste, plus pragmatique, mais aussi plus efficace rela-

17. Propos de Robert Barbault tirés de Frédéric Garlan, «Biodiversité: les associations, partenaires incontournables de la science ", dépêche AFP, 29 novembre 2006.

18. Ibidem.

19. Remarque issue d'entretiens avec Harold Levrel (chercheur, Ifremer) et Romain Julliard (chercheur, CRBPO).

20. Propos de Robert Barbault tirés de Frédéric Garlan, art. cit., op. cit.

21. Denis Buhot, «Classes moyennes et transformations d'un loisir de nature: le cas d'une association ornithologique », dans Anne Cadoret (dir.), Protection de la nature. Histoire et Idéologie, Paris, L'Harmattan, 1985. 
tivement au manque de moyens financiers: la «science participative», dont le succès (quoique jamais acquis définitivement) est rendu possible par l'existence d'un sentiment partagé de justice environnementale. Un tel sentiment, proposons-nous de montrer maintenant, émane de la valeur interactionnelle de l'environnement.

La forme de justice environnementale qui se dissimule derrière l'organisation du programme STOC semble se conformer à la notion de justice distributive telle qu'elle fut élaborée par John Rawls ${ }^{22}$. Restons toutefois conscients que la théorie de Rawls prend pleinement son sens en tant qu'analyse des libertés et des droits des citoyens au niveau de l'État, alors que l'exemple présent réduit la communauté contractualiste initiale à un réseau limité d'ornithologues ayant librement choisi de s'impliquer dans la conservation de populations d'oiseaux. La théorie de la justice comme équité donne à penser les structures procédurales de base des institutions sociales et politiques modernes. Outre un principe premier d'un droit intangible et égal à la liberté pour tout citoyen, cette théorie repose sur deux grands principes: un principe d' «égalité équitable des chances" - à talents donnés, tous les citoyens doivent jouir d'un égal accès aux positions sociales - et un principe dit de «différence» - les inégalités socio-économiques acquises doivent être telles qu'elles contribuent à améliorer de la meilleure manière qui soit la situation des moins avantagés.

Il n'est pas question ici de capital économique ou de biens fondamentaux, mais il est possible d'envisager les rapports entre les participants au programme STOC à partir de leurs échanges de capital symbolique et d'information en posant comme prémisse que la notion de justice environnementale doit être étendue jusqu'à l'inclusion des savoirs environnementaux.

La plaquette de présentation de Vigie-Nature affirme que «les participants ont un intérêt personnel à mener ces observations (par exemple documenter ce qui se passe à leur échelle) et pas seulement la motivation de participer à un grand projet national ${ }^{23}$ ”. Il est vrai que l'échange des connaissances entre les naturalistes participants, voire les simples citoyens volontaires, et les scientifiques du CRBPO qui récoltent, analysent, exploitent les données et communiquent les résultats,

22. John Rawls, Théorie de la justice [1971], Paris, Seuil, 1987.

23. Vigie-Nature: http://www.mnhn.fr/vigie-nature [consulté le 15 avril 2008]. 
permet à chacun d'y trouver son compte (y compris, in fine aux populations d'oiseaux menacées). Les scientifiques ont compris qu'il ne suffisait pas de faire appel à un moralisme abstrait et culpabilisateur pour mobiliser les naturalistes, au nom d'un supposé objectif commun de défense des espèces ${ }^{24}$. De toute évidence, instrumentalisée en tant que dispositif de relais du pouvoir des professionnels et des chercheurs, l'éthique environnementale demeurerait peu suivie et inefficace, quand bien même elle en appellerait à des valeurs et des fins partagées.

L'écologie participative ou citoyenne signe une forme de réconciliation entre l'écologie scientifique et le loisir ou l'engagement naturaliste et protectionniste par l'échange continu, juste et réciproque de «capital » symbolique ${ }^{25}$ contre du «capital » scientifique, le tout afin de préserver une sorte de «capital» naturel commun. Les scientifiques doivent faire acte de vulgarisation, d'écoute, de confiance ${ }^{26}$ et, en échange, ils recueillent des données, des idées, des problématiques nouvelles, qui leur servent de matière première pour leurs recherches. Les naturalistes consacrent du temps, de l'assiduité, font des efforts d'adaptation, font acte de propositions et de transparence et, en échange, ils recueillent de la considération (morale et scientifique), des éclaircissements et des analyses sur leur situation particulière qu'ils peuvent ensuite exploiter au niveau local.

Conformément au "principe de différence» de Rawls, il existe une inégalité de distribution du capital scientifique entre professionnels et amateurs quand bien même ces derniers seraient devenus des « experts profanes » fort savants sur les espèces et les écosystèmes locaux; mais il s'agit d'une forme d'inégalité assumée et rendue équitable par un ensemble de procédures démocratiques (forum, conférences, confrontation des points de vue, partage des données, reconnaissances des expériences propres à chaque acteur, circulation des savoirs, etc.) que l'on rassemble sous la bannière de la «démocratie technique»: c'est elle qui permet d'aller jusqu'à la «co-construction» d'outils scientifiques comme les protocoles de récolte de données, voire les indicateurs eux-mêmes. Ce processus de confrontation et de partage passe

24. Ce point a été souligné par Romain Julliard à propos des rapports plus anciens entre naturalistes et chercheurs.

25. Pierre, Bourdieu, Raisons pratiques, Paris, Seuil, 1994.

26. Ce qui passe en pratique par un abaissement des $\alpha$ côts $»$ de transaction et de communication, par l'accord préalable sur un vocabulaire commun par exemple (voir Harold Levrel, art. cit.). 
nécessairement par la construction d'une confiance partagée, condition nécessaire pour parvenir ultimement à de «bonnes » vérités. Le rapprochement est moins direct qu'en langue anglaise où le slogan «no truth without trust $t^{27}$ " exprime l'origine étymologique commune des deux concepts. Il n'empêche que la valeur interactionnelle qu'est la justice permet à l'éthique et à la science de s'entraîner l'une l'autre et de converger vers des finalités communes.

Mais il existe une autre forme de redistribution équitable des savoirs dans cette expérience de science participative: il s'agit aussi de ramener symboliquement chaque ornithologue, avec la nature diverse dont il se fait le porte-parole ${ }^{28}$, à un niveau considéré comme équitable pour tous. Certes, les scientifiques du CRBPO sont en contact privilégié avec des coordinateurs locaux qui relaient les informations auprès des observateurs, mais il n'est plus question de système hiérarchisé, voire élitiste, qui privilégiait et reconnaissait le seul travail des naturalistes amateurs les plus qualifiés et les plus actifs. Les données de chaque naturaliste, quels que soient par ailleurs son implication, son investissement (en ressources, en matériel, en temps), son ancienneté, ses connaissances, etc., sont toutes prises en compte sur un pied d'égalité.

De plus, en choisissant de s'appuyer sur 158 espèces communes d'oiseaux, le programme STOC privilégie ce que l'on appelle la nature ou biodiversité "ordinaire», et non les espèces rares ou exceptionnelles. Le programme STOC représente ainsi une sorte de lit de Procuste ornithologique qui réduit le poids des naturalistes des régions les plus favorisées pour la faune aviaire (les zones de marais, de montagne, les baies maritimes, etc.) au profit de tous les milieux (forêts, zones cultivées, zones urbaines, voire parkings de supermarché !).

De manière générale, le discours sur la biodiversité promu par le programme STOC fait prendre conscience de la valeur de toutes les espèces « sans grades", celles dites «communes», sans intérêt esthétique particulier, celles qu'on dit parfois encore «nuisibles» ou « invasives», et nous enseigne qu'à travers leurs fonctions écologiques, ce sont elles

27. Michael S. Carolan et Michael M. Bell, « In Truth we Trust: Discourse, Phenomenology, and the Social Relations of Knowledge in an Environmental Dispute ", Environmental Values, vol. 12, $\mathrm{n}^{\circ} 2$, 2003, p. 225-245.

28. Selon Bruno Latour, les scientifiques se font les «porte-parole» des entités (non-humains) qui peuplent le monde, tout comme les politiques se font les porte-parole des humains (voir Politiques de la nature, Paris, La Découverte, 1999). 
qui témoignent le mieux des changements de nos milieux, des menaces qui pèsent sur eux et sur les services qu'ils nous rendent du fait des assauts plus insistants du changement climatique ou de l'intensification des pratiques agricoles. Et s'il faut rappeler que «la détermination des effectifs de telle ou telle espèce est très directement fonction du nombre, de la compétence, mais surtout des attentes des observateurs ${ }^{29}$ ", l'un des mérites les plus saillants du programme STOC est d'avoir fait converger les attentes des observateurs en rendant justice à leur travail grâce à une reconnaissance équitable de leur engagement, quel que soit par ailleurs leur niveau initial de connaissance ou la valeur de l'environnement et des oiseaux dont ils se font les porte-parole.

\section{Conclusion}

Il semble difficile pour nombre d'écologistes de se défaire de cette opinion, presque un mythe, selon lequel «à moins d'être imposée par la force, $[\ldots]$ l'éthique environnementale [aurait] peu de chance de devenir une doctrine suffisamment partagée pour établir les contraintes nécessaires à la protection efficace de l'environnement ${ }^{30}$ ». Ce n'est pas la force pure du politique - accompagnée de ses excroissances fantasmatiques en termes de «fascisme vert » - qui résoudra la question cruciale de l'instanciation dans les actions individuelles et dans les politiques publiques des principes d'éthique environnementale. Doit-on dès lors se résigner à la seule dimension axiologique et normative de l'éthique? Non plus, car si l'éthique environnementale ne nécessite pas la force, elle requiert par contre la prise en compte de la dimension pragmatique dont les valeurs environnementales sont porteuses, et l'émergence des conditions de partage équitable des bénéfices cognitifs (ainsi qu'économiques, symboliques, sociaux, etc.) propres aux fins environnementales.

À cet égard, il peut paraître paradoxal que le champ de la recherche écologique soit l'un de ceux où le discours éthique a eu une influence parmi les plus importantes. Les scientifiques aiment en effet rappeler la

29. Denis Buhot, «Classes moyennes et transformations... ", art. cit., op. cit.

30. François Blais et Marcel Filion, «De l'éthique environnementale à l'écologie politique», Philosophiques, vol. 28, $\mathrm{n}^{\circ} 2,2001$. 
neutralité idéologique et morale de leur activité: ils devraient dévoiler ce qui est, révéler la vérité des faits, et laisser aux intellectuels et aux politiques la détermination de ce qui doit être. En réalité, c'est en se souciant d'intégrer à leur activité le champ des valeurs et des normes que les scientifiques découvrent de nouveaux faits et explorent de nouveaux horizons de la réalité. C'est comme si, dans leurs dimensions pragmatiques, le Bien et le Vrai se rejoignaient et se renforçaient mutuellement.

Finalement, on peut se demander si la recherche de vertus morales en écologie n'est malheureusement pas le symptôme d'une science peu assurée d'elle-même. Shrader-Frechette et $\mathrm{McCoy}^{31}$ affirment même que c'est à la sous-détermination théorique de l'écologie (autrement dit à ses lacunes en termes de structures nomologiques et théoriques propres aux sciences «dures») que ses hypothèses et ses méthodes peuvent être si aisément influencées par les valeurs de l'éthique environnementale. À moins que, tout simplement, cette sensibilité éthique ne témoigne de la maturité d'une science prenant pleinement conscience des enjeux sociaux immenses qu'elle est en passe d'affronter. 